\title{
A Quantitative Model for Bipolar Molecular Outflows
}

\author{
Zhi-Yun LI and Frank H. SHU \\ UC Berkeley
}

\begin{abstract}
.
Since their first discovery in 1980 , nearly 200 bipolar molecular outflows have now been observed. These outflows appear to be a ubiquitous phenomenon in the star formation process, although their origin remains unclear. Here, we adopt the thin-shell model of Shu, Ruden, Lada, \& Lizano (1991, ApJL, 370,31) in which bipolar molecular outflows are the swept-up ambient material by powerful winds from central young stellar objects. Besides explaining many observed features of bipolar sources, such as shape, velocity, and the typical extent, this model also naturally accounts for the so-called "Hubble law" of velocity field. However, it was criticized by Masson \& Chernin (1992, ApJ, 387, L47) on the ground that much less mass is observed at the high velocity end of some bipolar outflows than expected from this thin-shell model, unless the wind and/or the ambient medium are extremely anisotropic. We show that the extreme anisotropy is in fact inevitable both in the wind and in the ambient medium, because of the presence of dynamically important magnetic fields.

The wind is anisotropic because it is magneto-centrifugally driven, and the frozen-in toroidal field tends to collimate the flow toward the rotation axis. This effect is studied using the well-known "method of characteristics" and we conclude that a MHD wind always has two components: a well-collimated jet and a wind-angle wind. It is the wide-angle wind component that will interact with the ambient medium and produce bipolar molecular outflows. The ambient core medium is anisotropic because it arises from lower-density, magnetically-supported molecular clouds through ambipolar diffusion. Using self-similarity technique, we model the "pivotal state" of star formation, which divides the core formation phase and the core collapse (accretion) phase. We find that pivotal states have toroid-like density contours in general, with lower-density regions around the magnetic axis (where matter drains more easily).

When these anisotropies induced by magnetic fields are taken into account properly, the main argument against the wind-angle wind-driven, thin-shell model for bipolar molecular outflows disappears. Indeed, we show that the empirical mass-velocity relation found by Masson \& Chernin and others for bipolar outflows provides the best quantitative supporting evidence for the thin-shell theory!
\end{abstract}

